\title{
AN EXPONENTIAL INEQUALITY AND STRONG LIMIT THEOREMS FOR CONDITIONAL EXPECTATIONS
}

\author{
Alexey Chuprunov and István Fazekas
}

Department of Math. Stat. and Probability, Chebotarev Inst. of Mathematics and Mechanics, Kazan State University, Universitetskaya 17, 420008 Kazan, Russia, e-mail: achuprunov@mail.ru

Faculty of Informatics, University of Debrecen, P.O. Box 12, 4010 Debrecen, Hungary, e-mail: fazekasi@inf.unideb.hu, tel: 36-52-316666/22825

\begin{abstract}
An exponential inequality for the tail of the conditional expectation of sums of centered independent random variables is obtained. This inequality is applied to prove analogues of the Law of the Iterated Logarithm and the Strong Law of Large Numbers for conditional expectations. As corollaries we obtain certain strong theorems for the generalized allocation scheme and for the nonuniformly distributed allocation scheme.
\end{abstract}

\section{Introduction}

The generalized allocation scheme is widely studied (see [5], [6], [11]). The scheme contains several special cases such as the usual allocation scheme (see [13], [12], [7]) and the random forests (see [5], [11]).

In the papers [1] and [2] we obtained Strong Laws of Large Numbers (SLLN) for random allocations and for random forests, respectively. The proofs of those SLLN's were based on fourth moment inequalities (the moment inequalities were obtained by long direct calculations). Then we presented a unified approach to the above mentioned SLLN's ([3]). In the present paper we continue the study started in [3].

Consider the probability measure $\mathbb{P}$ and the conditional probability measure $\mathbb{P}^{A}$ with respect to the fixed event $A$. Let $\mathbb{E}^{A}$ denote the expectation with respect to $\mathbb{P}^{A}$. Let $\mu$ be the average of $N$ bounded independent random variables and let $\sigma^{2}$ be the average of the variances.

We prove the following exponential inequality for the tails

$$
\mathbb{P}^{A}\left\{\frac{\left|\mu-\mathbb{E}^{A} \mu\right|}{\sqrt{N}} \geq \varepsilon\right\} \leq \frac{\sqrt{2}}{\mathbb{P}(A)} e^{-\frac{\varepsilon^{2}}{16 \sigma^{2}}}(1+B),
$$

\footnotetext{
Key words and phrases: conditional expectation, moment, Khintchine's inequality, exponential inequality, generalized allocation scheme, Law of the Iterated Logarithm, Strong Law of Large Numbers.
}

2000 Mathematics Subject Classification: 60F15 Strong theorems. 
see Theorem 2.1, where $B$ depends on $\varepsilon$ and $\sigma^{2}$. The method of the proof of the exponential inequality is the following. We estimate the tail probability by the $p$-th moment of the sum of certain random variables which we majorize using Khintchine's inequality. Finally, we compute the minimum in $p$.

Using the above inequality, we prove some analogues of the Law of the Iterated Logarithm and the Strong Law of Large Numbers for the conditional expectations (Section 3).

Then the Law of the Iterated Logarithm and the Strong Laws of Large Numbers is applied to the generalized allocation scheme (Section 4). The random variables $\eta_{1}, \ldots, \eta_{N}$ satisfy a generalized allocation scheme, if equation (4.1) is satisfied with $\xi_{1}, \ldots, \xi_{N}$ having distribution (4.3).

Finally, in Section 5, we apply our general results to the allocations of balls into boxes having nonuniform probabilities.

Both in Section 4 and in Section 5 strong theorems are proved for $\mu_{s}=\mu_{s n N}$ being the number of boxes containing $s$ balls when $n$ balls are placed into $N$ boxes. We emphasize that the random variables $\mu_{s}=\mu_{s n N}$ depend on the number of boxes $(N)$ and the number of balls $(n)$, so that $n, N \rightarrow \infty$. Therefore we have proved strong limit results for a two-indexed sequence of random variables with indices varying in a sector (Theorem 4.2, Corollary 4.1, Theorem 5.1, Corollary 5.1).

\section{The main inequality}

In this section we prove exponential inequalities for the conditional tail probability of a sum of independent bounded random variables (Theorem 2.1).

Let $(\Omega, \mathcal{A}, \mathbb{P})$ denote the underlying probability space. Let $A \in \mathcal{A}$ be a fixed event such that $\mathbb{P}(A)>0$. Recall that the conditional probability $\mathbb{P}^{A}$ is defined by the formula

$$
\mathbb{P}^{A}(B)=\mathbb{P}(B \cap A) / \mathbb{P}(A), \quad B \in \mathcal{A} .
$$

We will denote by $\mathbb{E}^{A}$ the expectation with respect to the probability measure $\mathbb{P}^{A}$.

Let $\tau_{i}, 1 \leq i \leq N$, be nondegenerate independent random variables with variances $\sigma_{i}^{2}$, $1 \leq i \leq N$, and such that $\left|\tau_{i}\right| \leq C<\infty, 1 \leq i \leq N$, almost surely. Let $\left\{\tau_{i}^{\prime}: i=1, \ldots, N\right\}$ be an independent copy of $\left\{\tau_{i}: i=1, \ldots, N\right\}$. Denote $s_{i}$ the variance of $\left(\tau_{i}-\tau_{i}^{\prime}\right)^{2}$. Introduce the notation

$$
\sigma^{2}=\frac{1}{N} \sum_{i=1}^{N} \sigma_{i}^{2}, \quad s=\frac{1}{N} \sum_{i=1}^{N} s_{i}
$$

We will consider the random variable

$$
\mu=\sum_{i=1}^{N} \tau_{i}
$$

with respect to the probability $\mathbb{P}^{A}$ and also with respect to the probability $\mathbb{P}$.

The main result of the paper is the following. Let $Z$ denote a standard normal random variable. 
Theorem 2.1. (i) Let $\varepsilon \geq 4 \sqrt{2} \sigma$. Then we have

$$
\mathbb{P}^{A}\left\{\frac{\left|\mu-\mathbb{E}^{A} \mu\right|}{\sqrt{N}} \geq \varepsilon\right\} \leq \frac{\sqrt{2}}{\mathbb{P}(A)} e^{-\frac{\varepsilon^{2}}{16 \sigma^{2}}}(1+B)
$$

where

$$
B=B(N, \sigma)=\frac{s}{32}\left(\frac{\varepsilon^{2}}{8 \sigma^{4} \sqrt{N}}\right)^{2} f_{2}\left(\frac{2 \varepsilon^{2} C^{2}}{8 \sigma^{4} \sqrt{N}}\right)+\mathrm{O}\left(\frac{8 \sigma^{2}}{\varepsilon^{2}}\right)
$$

and

$$
f_{2}(x)=2 \mathbb{E}\left\{Z^{2} \exp (x|Z|)\right\}-1 .
$$

(ii) Let $\varepsilon \geq 4 \sqrt{2} \sigma$. Then we have

$$
\mathbb{P}^{A}\left\{\frac{\left|\mu-\mathbb{E}^{A} \mu\right|}{\sqrt{N}} \geq \varepsilon\right\} \leq \frac{\sqrt{2}}{\mathbb{P}(A)} e^{-\frac{\varepsilon^{2}}{16 \sigma^{2}}}\left(1+B_{3}\right)
$$

where

$$
B_{3}=\frac{s}{32}\left(\frac{\varepsilon^{2}}{8 \sigma^{4} \sqrt{N}}\right)^{2}+\frac{s C^{2}}{24}\left(\frac{\varepsilon^{2}}{8 \sigma^{4} \sqrt{N}}\right)^{3} f_{3}\left(2 C^{2} \frac{\varepsilon^{2}}{8 \sigma^{4} \sqrt{N}}\right)+\mathrm{O}\left(\frac{8 \sigma^{2}}{\varepsilon^{2}}\right)
$$

and

$$
f_{3}(x)=\mathbb{E}\left\{|Z|^{3} \exp (x|Z|)\right\} .
$$

Remark 2.1.

$$
\begin{gathered}
f_{2}(x)=4\left(e^{x^{2} / 2}\left(x^{2}+1\right) \Phi(x)+x / \sqrt{2 \pi}\right)-1, \\
f_{3}(x)=2 e^{x^{2} / 2}\left(x^{3}+3 x\right) \Phi(x)+2\left(x^{2}+2\right) / \sqrt{2 \pi},
\end{gathered}
$$

where $\Phi(x)=\int_{-\infty}^{x}(1 / \sqrt{2 \pi}) e^{-t^{2} / 2} d t$ is the standard normal cumulative distribution function.

In the proof of Theorem 2.1 we will use the following lemmas.

Lemma 2.1. Let $1 \leq p<\infty$ and let $S$ be a random variable. Then we have

$$
\mathbb{E}^{A}\left|S-\mathbb{E}^{A} S\right|^{p} \leq 2^{p} \frac{\mathbb{E}|S-\mathbb{E} S|^{p}}{\mathbb{P}(A)} .
$$

Inequality (2.8) was obtained in [3] with $4^{p}$ in place of $2^{p}$. The above form of the inequality was proved in [9].

Our study will be based on the following Khintchine type inequality.

Lemma 2.2. Let $1 \leq p<\infty$. Let $\zeta_{i}, 1 \leq i \leq N$, be independent centered random variables. Let $\left\{\zeta_{i}^{\prime}: 1 \leq i \leq N\right\}$ be an independent copy of $\left\{\zeta_{i}: 1 \leq i \leq N\right\}$. Then we have

$$
\mathbb{E}\left|\sum_{i=1}^{N} \zeta_{i}\right|^{p} \leq\left(B_{p}\right)^{p} \mathbb{E}\left|\sum_{i=1}^{N}\left(\zeta_{i}-\zeta_{i}^{\prime}\right)^{2}\right|^{p / 2}
$$


where $B_{p}=1$ if $0<p<2$ and $B_{p}=\left(\mathbb{E}|Z|^{p}\right)^{1 / p}$ if $2 \leq p<\infty$ with $Z$ being a Gaussian random variable with mean 0 and variance 1 .

If the $\zeta_{i}$ 's are symmetric, then we have

$$
\mathbb{E}\left|\sum_{i=1}^{N} \zeta_{i}\right|^{p} \leq\left(B_{p}\right)^{p} \mathbb{E}\left|\sum_{i=1}^{N}\left(\zeta_{i}\right)^{2}\right|^{p / 2}
$$

Proof. First recall that Khintchine's original inequality ([8]) has the following version (see [4]). Let $0<p<\infty$ be fixed and let $r_{i}, i \in \mathbb{N}$, be the Rademacher functions. Let $\mathbb{E}_{r}$ denote the expectation with respect to $\left\{r_{i}\right\}$. Then, for any $c_{i} \in \mathbb{R}, 1 \leq i \leq N$, we have

$$
\left(\mathbb{E}_{r}\left|\sum_{i=1}^{N} c_{i} r_{i}\right|^{p}\right)^{1 / p} \leq B_{p}\left(\sum_{i=1}^{N}\left(c_{i}\right)^{2}\right)^{1 / 2},
$$

where $B_{p}=1$, if $0<p<2$ and $B_{p}=\left(\mathbb{E}|Z|^{p}\right)^{1 / p}$, if $2 \leq p<\infty$.

Now we can assume that $\left\{r_{i}: 1 \leq i \leq N\right\}$ is independent of $\left\{\zeta_{i}^{\prime}: 1 \leq i \leq N\right\}$ and $\left\{\zeta_{i}: 1 \leq i \leq N\right\}$. Therefore $\left(\zeta_{i}-\zeta_{i}^{\prime}\right) r_{i}$ has the same distribution as $\zeta_{i}-\zeta_{i}^{\prime}$. By the Jensen inequality, the independence, and (2.11), we have

$$
\begin{gathered}
\mathbb{E}\left|\sum_{i=1}^{N}\left(\zeta_{i}-0\right)\right|^{p} \leq \mathbb{E}\left|\sum_{i=1}^{N}\left(\zeta_{i}-\zeta_{i}^{\prime}\right)\right|^{p}= \\
=\mathbb{E}_{r}\left|\sum_{i=1}^{N}\left(\zeta_{i}-\zeta_{i}^{\prime}\right) r_{i}\right|^{p} \leq\left(B_{p}\right)^{p} \mathbb{E}\left|\sum_{i=1}^{N}\left(\zeta_{i}-\zeta_{i}^{\prime}\right)^{2}\right|^{p / 2} .
\end{gathered}
$$

Lemma 2.3. Let $\zeta_{i}, 1 \leq i \leq N$, be independent random variables with expectations $a_{i}$ and variances $v_{i}^{2}$, and such that $\left|\zeta_{i}\right| \leq C<\infty$ almost surely, $1 \leq i \leq N$. Let

$$
a^{\prime}=\frac{1}{N} \sum_{i=1}^{N} a_{i}, \quad a=\left|a^{\prime}\right|, \quad v^{2}=\frac{1}{N} \sum_{i=1}^{N} v_{i}^{2} .
$$

Assume that $a \neq 0$.

(i) Let $p \geq 2$. Then we have

$$
a^{p} \leq \mathbb{E}\left|\frac{1}{N} \sum_{i=1}^{N} \zeta_{i}\right|^{p} \leq a^{p}(1+B)
$$

where

$$
B=\frac{v^{2}}{2} \frac{p(p-1)}{a^{2} N} f_{2}\left(\frac{p-2}{a \sqrt{N}} 2 C\right)
$$

and $f_{2}(x)$ is given by $(2.3)$.

(ii) Let $p \geq 3$. Then we have

$$
\begin{gathered}
a^{p}\left(1+\frac{v^{2}}{2} \frac{p(p-1)}{(a \sqrt{N})^{2}}-\frac{v^{2}}{3} \frac{p(p-1)(p-2)}{(a \sqrt{N})^{3}} 2 C f_{3}\left(\frac{p-3}{a \sqrt{N}} 2 C\right)\right) \leq \mathbb{E}\left|\frac{1}{N} \sum_{i=1}^{N} \zeta_{i}\right|^{p} \leq \\
\leq a^{p}\left(1+\frac{v^{2}}{2} \frac{p(p-1)}{(a \sqrt{N})^{2}}+\frac{v^{2}}{3} \frac{p(p-1)(p-2)}{(a \sqrt{N})^{3}} 2 C f_{3}\left(\frac{p-3}{a \sqrt{N}} 2 C\right)\right)
\end{gathered}
$$

where $f_{3}(x)$ is given by (2.5). 
Proof. (i) The left hand side of (2.12) follows from Jensen's inequality. Let $\left\{\zeta_{i}^{\prime}: 1 \leq i \leq N\right\}$ be an independent copy of $\left\{\zeta_{i}: 1 \leq i \leq N\right\}$. Since $p \geq 2$, the function $g(x)=\left|a^{\prime}+x\right|^{p}$ has a continuous second derivative. By Taylor's expansion, it holds that

$$
g(x)=a^{p}+\operatorname{sgn}\left(a^{\prime}\right) \frac{p a^{p-1}}{1 !} x+\frac{p(p-1)\left|a^{\prime}+\theta x\right|^{p-2}}{2 !} x^{2},
$$

where $\theta \in(-1,1)$. Therefore, with $x=\sum_{i=1}^{N}\left(\zeta_{i}-a_{i}\right) / N$, we obtain

$$
\begin{gathered}
\mathbb{E}\left|\frac{1}{N} \sum_{i=1}^{N} \zeta_{i}\right|^{p}=a^{p}+\frac{p(p-1)}{2} \mathbb{E}\left\{\left|a^{\prime}+\frac{\theta}{N} \sum_{i=1}^{N}\left(\zeta_{i}-a_{i}\right)\right|^{p-2}\left(\frac{1}{N} \sum_{i=1}^{N}\left(\zeta_{i}-a_{i}\right)\right)^{2}\right\}= \\
=a^{p}\left(1+B^{\prime}\right),
\end{gathered}
$$

where $\theta=\theta(\omega)$ with $-1 \leq \theta \leq 1$. Since $1+x \leq e^{x}, x \in \mathbb{R}$, moreover

$$
\mathbb{E}\left|\frac{1}{N} \sum_{i=1}^{N}\left(\zeta_{i}-\zeta_{i}^{\prime}\right)^{2}\right|^{\frac{k+2}{2}} \leq \mathbb{E}\left(\frac{1}{N} \sum_{i=1}^{N}\left(\zeta_{i}-\zeta_{i}^{\prime}\right)^{2}\right)(2 C)^{k}=2 v^{2}(2 C)^{k},
$$

therefore, using Khintchine's inequality (2.9), we obtain

$$
\begin{gathered}
B^{\prime} \leq \frac{p(p-1)}{2} \mathbb{E}\left\{\left(1+\left|\frac{\sum_{i=1}^{N}\left(\zeta_{i}-a_{i}\right)}{N a}\right|\right)^{p-2}\left(\frac{\sum_{i=1}^{N}\left(\zeta_{i}-a_{i}\right)}{N a}\right)^{2}\right\} \leq \\
\leq \frac{p(p-1)}{2} \mathbb{E}\left\{\exp \left((p-2)\left|\frac{\sum_{i=1}^{N}\left(\zeta_{i}-a_{i}\right)}{N a}\right|\right)\left(\frac{\sum_{i=1}^{N}\left(\zeta_{i}-a_{i}\right)}{N a}\right)^{2}\right\}= \\
=\frac{p(p-1)}{2} \sum_{k=0}^{\infty} \frac{1}{k !}(p-2)^{k} \mathbb{E}\left|\frac{\sum_{i=1}^{N}\left(\zeta_{i}-a_{i}\right)}{N a}\right|^{k+2} \leq \\
\leq \frac{1}{2} \frac{p(p-1)}{a^{2} N} \sum_{k=0}^{\infty} \frac{1}{k !}\left(\frac{p-2}{a \sqrt{N}}\right)^{k} \mathbb{E}\left|\frac{\sum_{i=1}^{N}\left(\zeta_{i}-a_{i}\right)}{\sqrt{N}}\right|^{k+2} \leq \\
\leq \frac{1}{2} \frac{p(p-1)}{a^{2} N}\left(v^{2}+\sum_{k=1}^{\infty} \frac{1}{k !}\left(\frac{p-2}{a \sqrt{N}}\right)^{k} \mathbb{E}|Z|^{k+2} \mathbb{E}\left|\frac{\sum_{i=1}^{N}\left(\zeta_{i}-\zeta_{i}^{\prime}\right)^{2}}{N}\right|^{\frac{k+2}{2}}\right) \leq \\
\leq \frac{v^{2}}{2} \frac{p(p-1)}{a^{2} N}\left(1+2 \sum_{k=1}^{\infty} \frac{1}{k !}\left(\frac{p-2}{a \sqrt{N}}\right)^{k} \mathbb{E}|Z|^{k+2}(2 C)^{k}\right)= \\
=\frac{v^{2}}{2} \frac{p(p-1)}{a^{2} N}\left(1+2\left(\mathbb{E}\left(Z^{2} \exp \left(\frac{p-2}{a \sqrt{N}} 2 C|Z|\right)\right)-1\right)\right)=\frac{v^{2}}{2} \frac{p(p-1)}{a^{2} N} f_{2}\left(\frac{p-2}{a \sqrt{N}} 2 C\right)=B .
\end{gathered}
$$

The proof of (i) is complete. 
(ii) Now $p \geq 3$. Since $g(x)=\left|a^{\prime}+x\right|^{p}$ has a continuous third derivative, by Taylor's expansion, we have

$$
g(x)=a^{p}+\frac{\operatorname{sgn}\left(a^{\prime}\right) p a^{p-1}}{1 !} x+\frac{p(p-1) a^{p-2}}{2 !} x^{2}+\frac{\operatorname{sgn}\left(a^{\prime}+\theta x\right) p(p-1)(p-2)\left|a^{\prime}+\theta x\right|^{p-3}}{3 !} x^{3}
$$

where $\theta \in(-1,1)$. Therefore, with $x=\sum_{i=1}^{N}\left(\zeta_{i}-a_{i}\right) / N$, we obtain

$$
\begin{gathered}
\mathbb{E}\left|\frac{1}{N} \sum_{i=1}^{N} \zeta_{i}\right|^{p}=a^{p}+\frac{p(p-1)}{2 N} a^{p-2} v^{2}+ \\
+\frac{p(p-1)(p-2)}{6} \mathbb{E}\left\{S\left|a^{\prime}+\theta \frac{1}{N} \sum_{i=1}^{N}\left(\zeta_{i}-a_{i}\right)\right|^{p-3}\left(\frac{1}{N} \sum_{i=1}^{N}\left(\zeta_{i}-a_{i}\right)\right)^{3}\right\}= \\
=a^{p}\left(1+\frac{p(p-1)}{2(a \sqrt{N})^{2}} v^{2}+B^{\prime}\right)
\end{gathered}
$$

where $\theta=\theta(\omega)$ with $-1 \leq \theta \leq 1$ and $S=\operatorname{sgn}\left\{a^{\prime}+\theta \sum_{i=1}^{N}\left(\zeta_{i}-a_{i}\right) / N\right\}$. Since $1+x \leq e^{x}$, $x \in \mathbb{R}$, by (2.14) and Khintchine's inequality (2.9), we obtain

$$
\begin{gathered}
\left|B^{\prime}\right| \leq \frac{p(p-1)(p-2)}{6} \mathbb{E}\left\{\left(1+\left|\frac{1}{N a} \sum_{i=1}^{N}\left(\zeta_{i}-a_{i}\right)\right|\right)^{p-3}\left|\frac{1}{N a} \sum_{i=1}^{N}\left(\zeta_{i}-a_{i}\right)\right|^{3}\right\} \leq \\
\leq \frac{p(p-1)(p-2)}{6} \mathbb{E}\left\{\exp \left((p-3)\left|\frac{1}{N a} \sum_{i=1}^{N}\left(\zeta_{i}-a_{i}\right)\right|\right)\left|\frac{1}{N a} \sum_{i=1}^{N}\left(\zeta_{i}-a_{i}\right)\right|^{3}\right\} \leq \\
\leq \frac{p(p-1)(p-2)}{6} \sum_{k=0}^{\infty} \frac{1}{k !}(p-3)^{k} \mathbb{E}\left|\frac{\sum_{i=1}^{N}\left(\zeta_{i}-a_{i}\right)}{N a}\right|^{k+3} \leq \\
\leq \frac{p(p-1)(p-2)}{6(a \sqrt{N})^{3}} \sum_{k=0}^{\infty} \frac{1}{k !}\left(\frac{p-3}{a \sqrt{N}}\right)^{k} \mathbb{E}\left|\frac{\sum_{i=1}^{N}\left(\zeta_{i}-a_{i}\right)}{\sqrt{N}}\right|^{k+3} \leq \\
\leq \frac{p(p-1)(p-2)}{6(a \sqrt{N})^{3}} \sum_{k=0}^{\infty} \frac{1}{k !}\left(\frac{p-3}{a \sqrt{N}}\right)^{k} \mathbf{E}|Z|^{k+3} \mathbb{E}\left|\frac{\sum_{i=1}^{N}\left(\zeta_{i}-\zeta_{i}^{\prime}\right)^{2}}{N}\right|^{\frac{k+3}{2}} \leq \\
\leq \frac{p(p-1)(p-2)}{6(a \sqrt{N})^{3}} 2 v^{2} 2 C \sum_{k=0}^{\infty} \frac{1}{k !}\left(\frac{p-3}{a \sqrt{N}}\right)^{k} \mathbb{E}|Z|^{k+3}(2 C)^{k}= \\
\leq \frac{p(p-1)(p-2)}{6(a \sqrt{N})^{3}} 2 v^{2} 2 C \mathbb{E}\left(|Z|^{3} \exp \left(\frac{p-3}{a \sqrt{N}} 2 C|Z|\right)\right)= \\
=\frac{v^{2}}{3} \frac{p(p-1)(p-2)}{(a \sqrt{N})^{3}} 2 C f_{3}\left(\frac{p-3}{a \sqrt{N}} 2 C\right) .
\end{gathered}
$$


We have seen that

$$
a^{p}\left(1+\frac{p(p-1)}{2(a \sqrt{N})^{2}} v^{2}-\left|B^{\prime}\right|\right) \leq \mathbb{E}\left|\frac{1}{N} \sum_{i=1}^{N} \zeta_{i}\right|^{p} \leq a^{p}\left(1+\frac{p(p-1)}{2(a \sqrt{N})^{2}} v^{2}+\left|B^{\prime}\right|\right) .
$$

Therefore (2.15) implies (2.13). The proof of (ii) is complete.

Proof of Theorem 2.1. It is known that $\mathbb{E}|Z|^{p}=\left(2^{\frac{p}{2}} / \sqrt{\pi}\right) \Gamma\left(\frac{p}{2}+\frac{1}{2}\right)$. Using [10], we have

$$
\begin{gathered}
\mathbb{E}|Z|^{p}=\frac{2^{\frac{p}{2}}}{\sqrt{\pi}} \Gamma\left(\frac{p}{2}+\frac{1}{2}\right)=\frac{2^{\frac{p}{2}}}{\sqrt{\pi}} e^{-\frac{p}{2}-\frac{1}{2}}\left(\frac{p}{2}+\frac{1}{2}\right)^{\frac{p}{2}+\frac{1}{2}} \sqrt{\frac{2 \pi}{\left(\frac{p}{2}+\frac{1}{2}\right)}}\left(1+\mathrm{O}\left(\frac{1}{p}\right)\right)= \\
=\sqrt{2} 2^{\frac{p}{2}} e^{-\frac{p}{2}-\frac{1}{2}}\left(\frac{p}{2}+\frac{1}{2}\right)^{\frac{p}{2}}\left(1+\mathrm{O}\left(\frac{1}{p}\right)\right)=\sqrt{2} e^{-\frac{p}{2}} e^{-\frac{1}{2}}(p+1)^{\frac{p}{2}}\left(1+\mathrm{O}\left(\frac{1}{p}\right)\right)= \\
=\sqrt{2} e^{-\frac{p}{2}} e^{-\frac{1}{2}}\left(\frac{p+1}{p}\right)^{\frac{p}{2}} p^{\frac{p}{2}}\left(1+\mathrm{O}\left(\frac{1}{p}\right)\right) \leq \sqrt{2} e^{-\frac{p}{2}} p^{\frac{p}{2}}\left(1+\mathrm{O}\left(\frac{1}{p}\right)\right) .
\end{gathered}
$$

Therefore, using Markov's inequality, Lemma 2.1, Khintchine's inequality (2.9) for $p / 2 \geq 2$, and (2.12), we obtain

$$
\begin{gathered}
\mathbb{P}^{A}\left\{\frac{\left|\mu-\mathbb{E}^{A} \mu\right|}{\sqrt{N}} \geq \varepsilon\right\} \leq \frac{1}{\varepsilon^{p}} \mathbb{E}^{A}\left(\frac{\left|\mu-\mathbb{E}^{A} \mu\right|}{\sqrt{N}}\right)^{p} \leq \\
\leq \frac{2^{p}}{\varepsilon^{p} \mathbb{P}(A)} \mathbb{E}\left(\frac{|\mu-\mathbb{E} \mu|}{\sqrt{N}}\right)^{p} \leq \sqrt{2} \frac{2^{p} e^{-\frac{p}{2}} p^{p / 2}}{\varepsilon^{p} \mathbb{P}(A)} \mathbb{E}\left|\frac{\sum_{i=1}^{N}\left(\tau_{i}-\tau_{i}^{\prime}\right)^{2}}{N}\right|^{p / 2}\left(1+\mathrm{O}\left(\frac{1}{p}\right)\right) \leq \\
\leq \sqrt{2} \frac{2^{p} e^{-\frac{p}{2}} p^{p / 2}}{\varepsilon^{p} \mathbb{P}(A)}\left(2 \sigma^{2}\right)^{p / 2}\left(1+\frac{s}{2}\left(\frac{p}{4 \sigma^{2} \sqrt{N}}\right)^{2} f_{2}\left(\frac{p}{4 \sigma^{2} \sqrt{N}} 8 C^{2}\right)\right)\left(1+\mathrm{O}\left(\frac{1}{p}\right)\right) .
\end{gathered}
$$

For $p=\frac{\varepsilon^{2}}{8 \sigma^{2}}$ from (2.17) we obtain (2.1), i.e.

$$
\mathbb{P}^{A}\left\{\frac{\left|\mu-\mathbb{E}^{A} \mu\right|}{\sqrt{N}} \geq \varepsilon\right\} \leq e^{-\frac{\varepsilon^{2}}{16 \sigma^{2}}} \frac{\sqrt{2}}{\mathbb{P}(A)}\left(1+\frac{s}{8}\left(\frac{\varepsilon^{2}}{16 \sigma^{4} \sqrt{N}}\right)^{2} f_{2}\left(\frac{\varepsilon^{2} C^{2}}{4 \sigma^{4} \sqrt{N}}\right)\right)\left(1+\mathrm{O}\left(\frac{8 \sigma^{2}}{\varepsilon^{2}}\right)\right) .
$$

To prove (2.4), apply (2.13) for the right hand side of (2.16). Then we obtain $\mathbb{P}^{A}\left\{\frac{\left|\mu-\mathbb{E}^{A} \mu\right|}{\sqrt{N}} \geq \varepsilon\right\} \leq \frac{\sqrt{2}}{\mathbb{P}(A)} e^{-p / 2}\left(\frac{8 p \sigma^{2}}{\varepsilon^{2}}\right)^{p / 2}\left(1+\frac{p^{2} s}{8\left(2 \sigma^{2} \sqrt{N}\right)^{2}}+\frac{p^{3} s C^{2}}{3\left(2 \sigma^{2} \sqrt{N}\right)^{3}} f_{3}\left(\frac{p 2 C^{2}}{\sigma^{2} \sqrt{N}}\right)\right)$.

Then put $p=\frac{\varepsilon^{2}}{8 \sigma^{2}}$. The proof is complete. 


\section{Strong limit theorems}

In this section we apply the exponential inequalities of Section 2 to describe the almost sure asymptotic behaviour of sums of certain non-independent random variables.

Assume that all random variables are defined on the probability space $(\Omega, \mathcal{A}, \mathbb{P})$. The conditional distribution of the stochastic element $\eta$ with respect to the fixed event $A$ is defined as $\mathbb{P}(\eta \in B \mid A), B \in \mathcal{A}$. We denote it by $(\eta \mid A)$.

In this section we study the following model. Consider the strictly increasing sequence of integer numbers $N_{k}, k \in \mathbb{N}$, and the sequence of the events $A=A(k) \in \mathcal{A}, k \in \mathbb{N}$, having property

$$
\mathbb{P}(A(k)) \geq C^{\prime \prime} / N_{k}^{\beta}
$$

for some $\beta>0, C^{\prime \prime}>0$. For each fixed $k$, let $\xi_{k i}, 1 \leq i \leq N_{k}$, be independent random variables with variances $\sigma_{k i}^{2}$, and such that $\left|\xi_{k i}\right| \leq C_{0}<\infty$ almost surely, $1 \leq i \leq N_{k}$. Suppose that the family $\left\{\eta_{k i}: 1 \leq i \leq N_{k}\right\}$ has the same distribution as the conditional distribution $\left\{\left(\xi_{k i} \mid A(k)\right): 1 \leq i \leq N_{k}\right\}$. We emphasize that the random variables $\eta_{k 1}, \ldots, \eta_{k N_{k}}$ can be dependent. Let

$$
\sigma_{k}^{2}=\frac{1}{N_{k}} \sum_{i=1}^{N_{k}} \sigma_{k i}^{2}
$$

Assume that there exists $0<C_{1}<\infty$ such that

$$
\frac{\ln \left(N_{k}\right)}{\sigma_{k}^{2} \sqrt{N_{k}}} \leq C_{1} \text { for all } k \in \mathbb{N} \text {. }
$$

Consider the sums of random variables

$$
S_{k}=\sum_{i=1}^{N_{k}} \eta_{k i}
$$

Now we will prove the following analogue of the Law of the Iterated Logarithm.

Theorem 3.1. Suppose that (3.1) and (3.2) are valid for the above defined model. Then we have

$$
\limsup _{k \rightarrow \infty} \frac{\left|S_{k}-\mathbb{E} S_{k}\right|}{\sqrt{N_{k} \ln \left(N_{k}\right)} \sigma_{k}} \leq 4 \sqrt{\beta+1} \quad \text { almost surely. }
$$

Proof. Let $S_{k}^{\prime}=\sum_{i=1}^{N_{k}} \xi_{k i}, k=1,2, \ldots$ By Theorem 2.1 we have

$$
\begin{gathered}
\sum_{k=1}^{\infty} \mathbb{P}\left\{\frac{\left|S_{k}-\mathbb{E} S_{k}\right|}{\sqrt{N_{k} \ln \left(N_{k}\right)} \sigma_{k}} \geq t\right\}=\sum_{k=1}^{\infty} \mathbb{P}\left\{\frac{\left|S_{k}-\mathbb{E} S_{k}\right|}{\sqrt{N_{k}}} \geq \sigma_{k} \sqrt{\ln \left(N_{k}\right)} t\right\}= \\
=\sum_{k=1}^{\infty} \mathbb{P}^{A(k)}\left\{\frac{\left|S_{k}^{\prime}-\mathbb{E}^{A(k)} S_{k}^{\prime}\right|}{\sqrt{N_{k}}} \geq \sigma_{k} \sqrt{\ln \left(N_{k}\right)} t\right\} \leq \sum_{k=1}^{\infty} \frac{\sqrt{2}}{P(A(k))} e^{-\frac{\ln \left(N_{k}\right) t^{2} \sigma_{k}^{2}}{16 \sigma_{k}^{2}}}\left(1+B\left(N_{k}, \sigma_{k}\right)\right)
\end{gathered}
$$


where $B(N, \sigma)$ is defined by $(2.2)$. Therefore

$$
B\left(N_{k}, \sigma_{k}\right)=\frac{s}{8}\left(\frac{t^{2} \ln \left(N_{k}\right)}{16 \sigma_{k}^{2} \sqrt{N_{k}}}\right) f_{2}\left(\frac{t^{2} \ln \left(N_{k}\right) C^{2}}{4 \sigma_{k}^{2} \sqrt{N_{k}}}\right) \mathrm{O}\left(\frac{8}{t^{2} \ln \left(N_{k}\right)}\right) .
$$

As $\left|\xi_{i k}\right| \leq C_{0}$, therefore $s$ is bounded, so, by $(3.2),\left\{B\left(N_{k}, \sigma_{k}\right)\right\}$ is a bounded sequence. Now, by $(3.1)$,

$$
\sum_{k=1}^{\infty} \mathbb{P}\left\{\frac{\left|S_{k}-\mathbb{E} S_{k}\right|}{\sqrt{N_{k} \ln \left(N_{k}\right)} \sigma_{k}} \geq t\right\} \leq K \sum_{k=1}^{\infty} N_{k}^{\beta-t^{2} / 16}
$$

It is finite when $t>4 \sqrt{\beta+1}$. Consequently, by the Borel-Cantelli lemma, we have $\limsup _{k \rightarrow \infty} \frac{\left|S_{k}-\mathbb{E} S_{k}\right|}{\sqrt{N_{k} \ln \left(N_{k}\right)} \sigma_{0 k}} \leq t$ almost surely. This implies (3.3).

As corollaries of Theorem 3.1, we obtain the following laws of large numbers.

Corollary 3.1. Suppose that (3.1) and (3.2) are valid for the model defined in this section. Let $d_{k}, k \in \mathbb{N}$, be a sequence such that $\sigma_{k} \sqrt{N_{k} \ln \left(N_{k}\right)} / d_{k} \rightarrow 0$, as $k \rightarrow \infty$. Then we have

$$
\lim _{k \rightarrow \infty} \frac{S_{k}-\mathbb{E} S_{k}}{d_{k}}=0 \quad \text { almost surely. }
$$

Proof. Observe that

$$
\frac{S_{k}-\mathbb{E} S_{k}}{d_{k}}=\frac{\sigma_{k} \sqrt{N_{k} \ln \left(N_{k}\right)}}{d_{k}} \frac{S_{k}-\mathbb{E} S_{k}}{\sigma_{k} \sqrt{N_{k} \ln \left(N_{k}\right)}} .
$$

By Theorem 3.1, $\frac{S_{k}-\mathbb{E} S_{k}}{\sigma_{k} \sqrt{N_{k} \ln \left(N_{k}\right)}}$ is an almost surely bounded sequence. This implies the corollary.

Corollary 3.2. Suppose that (3.1) and (3.2) are valid for the model defined in this section. Let $d_{k}, k \in \mathbb{N}$, be a sequence such that $\sigma_{k} \sqrt{N_{k} \ln \left(N_{k}\right)} / d_{k} \rightarrow 0$ and $\mathbb{E} S_{k} / d_{k} \rightarrow$ a as $k \rightarrow \infty$. Then we have

$$
\lim _{k \rightarrow \infty} \frac{S_{k}}{d_{k}}=a \quad \text { almost surely. }
$$

Proof. Since

$$
\frac{S_{k}}{d_{k}}=\frac{S_{k}-\mathbb{E} S_{k}}{d_{k}}+\frac{\mathbb{E} S_{k}}{d_{k}}
$$

the result follows from Corollary 3.1.

\section{Application to the generalized allocation scheme}

In the generalized scheme of allocations of particles into cells, the distribution of the cell contents is represented as the conditional distribution of independent random variables under 
the condition that their sum is fixed, see [5], [11]. We can describe the generalized allocation scheme as follows. Let $\eta_{1}, \ldots, \eta_{N}$ be nonnegative integer-valued random variables. They are considered as certain numerical characteristics of the combinatorial structure of $n$ elements consisting of $N$ components such that $\eta_{1}+\cdots+\eta_{N}=n$. If there exist independent random variables $\xi_{1}, \ldots, \xi_{N}$ such that the joint distribution of $\eta_{1}, \ldots, \eta_{N}$ admits the representation

$$
\mathbb{P}\left\{\eta_{1}=k_{1}, \ldots, \eta_{N}=k_{N}\right\}=\mathbb{P}\left\{\xi_{1}=k_{1}, \ldots, \xi_{N}=k_{N} \mid \xi_{1}+\cdots+\xi_{N}=n\right\},
$$

where $k_{1}, \ldots, k_{N}$ are arbitrary nonnegative integers such that $k_{1}+k_{2}+\cdots+k_{N}=n$, then we say that $\eta_{1}, \ldots, \eta_{N}$ obey a generalized allocation scheme with parameters $n$ and $N$, and independent random variables $\xi_{1}, \ldots, \xi_{N}$. Throughout this section we assume that the random variables $\eta_{1}, \ldots, \eta_{N}$ and $\xi_{1}, \ldots, \xi_{N}$ satisfy (4.1). When $\xi_{1}, \ldots, \xi_{N}$ are i.i.d. Poissonian random variables, then the vector $\eta_{1}, \ldots, \eta_{N}$ describes the usual allocation of $n$ balls into $N$ boxes. Details of the theory of generalized allocation schemes can be found in [5], [11].

In view of independence of the random variables $\xi_{1}, \ldots, \xi_{N}$, the study of several questions of the generalized allocation scheme can be reduced to problems of sums of independent random variables.

Let the random variables $\xi_{1}, \ldots, \xi_{N}$ be identically distributed. Usually (see, e.g., [6]) the random variables $\xi_{1}, \ldots, \xi_{N}$ are distributed as follows: $q_{k}=\mathbb{P}\left\{\xi_{1}=k\right\}=\left(b_{k} \theta^{k}\right) /(k ! B(\theta))$ where $b_{0}, b_{1}, \ldots$ is a certain sequence of non-negative numbers, and $B(\theta)=\sum_{k=0}^{\infty} b_{k} \theta^{k} / k$ !.

Therefore, in this section, we consider a sequence of non-negative numbers $b_{0}, b_{1}, \ldots$ with $b_{0}>0, b_{1}>0$ and assume that the convergence radius $R$ of the series

$$
B(\theta)=\sum_{k=0}^{\infty} \frac{b_{k} \theta^{k}}{k !}
$$

is positive. Let us introduce the integer-valued random variable $\xi=\xi(\theta)$ (where $\theta>0$ ) with distribution

$$
q_{k}=q_{k}(\theta)=\mathbb{P}\{\xi=k\}=\frac{b_{k} \theta^{k}}{k ! B(\theta)}, k=0,1,2, \ldots
$$

By [6], one has

$$
m=m(\theta)=\mathbb{E} \xi=\frac{\theta B^{\prime}(\theta)}{B(\theta)}
$$

and

$$
\sigma^{2}=\sigma^{2}(\theta)=\mathbb{D}^{2} \xi=\frac{\theta^{2} B^{\prime \prime}(\theta)}{B(\theta)}+\frac{\theta B^{\prime}(\theta)}{B(\theta)}-\frac{\theta^{2}\left(B^{\prime}(\theta)\right)^{2}}{(B(\theta))^{2}} .
$$

The last equality implies that

$$
\sigma^{2}(\theta)=\theta m^{\prime}(\theta)
$$

Let $0<\theta^{\prime}<\theta^{\prime \prime}<R$. If $\sigma^{2}(\theta)=0$ for some $\theta \in\left[\theta^{\prime}, \theta^{\prime \prime}\right]$, then the random variable $\xi(\theta)$ is a constant. Since $b_{0}>0, b_{1}>0$, the random variable $\xi(\theta)$ is not a constant. Therefore $\sigma^{2}(\theta)$, $\theta \in\left[\theta^{\prime}, \theta^{\prime \prime}\right]$ is a positive continuous function. Consequently,

$$
0<C_{1}=\inf _{\theta \in\left[\theta^{\prime}, \theta^{\prime \prime}\right]} \sigma^{2}(\theta) \leq \sup _{\theta \in\left[\theta^{\prime}, \theta^{\prime \prime}\right]} \sigma^{2}(\theta)=C_{2}<\infty
$$


By (4.5) and (4.6), we have

$$
0<\frac{C_{1}}{\theta^{\prime \prime}}=\inf _{\theta \in\left[\theta^{\prime}, \theta^{\prime \prime}\right]} m^{\prime}(\theta) \leq \sup _{\theta \in\left[\theta^{\prime}, \theta^{\prime \prime}\right]} m^{\prime}(\theta)=\frac{C_{2}}{\theta^{\prime}}<\infty .
$$

Since $m^{\prime}(\theta)>0$, then $m(\theta), \theta \in\left[\theta^{\prime}, \theta^{\prime \prime}\right]$, is a positive, continuous, strictly increasing function. We will denote by $m^{-1}$ the inverse function of $m$.

We see that the random variable $\xi(\theta)$ has all moments, if $\theta<R$.

Throughout this section, let $\xi_{1}=\xi_{1}(\theta), \ldots, \xi_{N}=\xi_{N}(\theta)$ be independent copies of $\xi(\theta)$ where $\xi=\xi(\theta)$ has distribution (4.3). Let $\eta_{1}=\eta_{1}(\theta), \ldots, \eta_{N}=\eta_{N}(\theta)$ satisfy (4.1) with $\xi_{1}=\xi_{1}(\theta), \ldots, \xi_{N}=\xi_{N}(\theta)$. So $\eta_{1}, \ldots, \eta_{N}$ also depend on $\theta$. Introduce notation $\alpha=n / N$. Let $\theta_{\alpha}=m^{-1}(\alpha)$. So in what follows the parameter $\theta=\theta_{\alpha}=m^{-1}(\alpha)$ will depend on $\alpha=n / N$.

Consider the event

$$
A=A_{N}(n)=\left\{\omega \in \Omega: \xi_{1}\left(\theta_{\alpha}\right)(\omega)+\xi_{2}\left(\theta_{\alpha}\right)(\omega)+\cdots+\xi_{N}\left(\theta_{\alpha}\right)(\omega)=n\right\}
$$

where $\theta_{\alpha}=m^{-1}(\alpha)$. Let $P_{N}(n)=\mathbb{P}\left(A_{N}(n)\right)$.

Lemma 4.1. (See [3].) Let $0<\alpha^{\prime}<\alpha^{\prime \prime}$ be such that $m^{-1}\left(\alpha^{\prime \prime}\right)<R$. Let $\alpha=n / N$. Then there exists $N_{0} \in \mathbb{N}$ with the following property: if $n, N \in \mathbb{N}$ are such that $N>N_{0}$ and $\alpha^{\prime} \leq \alpha \leq \alpha^{\prime \prime}$, then we have

$$
P_{N}(n)>\frac{1}{4 \sigma\left(\theta_{\alpha}\right) \sqrt{N}}
$$

Let $\sigma_{t \alpha}^{2}=q_{t}\left(\theta_{\alpha}\right)\left(1-q_{t}\left(\theta_{\alpha}\right)\right)$. We see that $\sigma_{t \alpha}^{2}$ is the variance of the indicator $\mathbb{I}_{\left\{\xi\left(\theta_{\alpha}\right)=t\right\}}$.

First consider a sequence of generalized allocations of $n_{k}$ balls into $N_{k}$ boxes such that $N_{k}<N_{k+1}, k \in \mathbb{N}$. Let $\alpha_{k}=n_{k} / N_{k}, \theta_{k}=m^{-1}\left(\alpha_{k}\right), \sigma_{t k}^{2}=\sigma_{t \alpha_{k}}^{2}$ and

$$
\mu_{t k}=\sum_{i=1}^{N_{k}} \mathbb{I}_{\left\{\eta_{i}=t\right\}}
$$

$\mu_{t k}$ can be considered as the number of boxes containing $t$ balls. We remark that if $b_{t}=0$, then $\mu_{t k}=0$. So we can concentrate on the case $b_{t}>0$. Now we will prove two analogues of the Law of the Iterated Logarithm for $\mu_{t k}$.

Theorem 4.1. Let $0<\alpha^{\prime}<\alpha^{\prime \prime}$ be such that $m^{-1}\left(\alpha^{\prime \prime}\right)<R$. Suppose that $0<\alpha^{\prime} \leq \alpha_{k} \leq \alpha^{\prime \prime}$ for all $k \in \mathbb{N}$. Let $t$ be fixed and assume that $b_{t}>0$. Then we have

$$
\limsup _{k \rightarrow \infty} \frac{\left|\mu_{t k}-\mathbb{E} \mu_{t k}\right|}{\sqrt{N_{k} \ln \left(N_{k}\right)} \sigma_{t k}} \leq 4 \sqrt{3 / 2} \text { almost surely. }
$$

Proof. By Lemma 4.1 and relation (4.6), we have

$$
P\left(A_{N_{k}}\left(n_{k}\right)\right)>\frac{1}{4 \sigma\left(\theta_{k}\right) \sqrt{N_{k}}} \geq \frac{C^{\prime \prime}}{N_{k}^{1 / 2}} .
$$


As $C^{\prime \prime}>0$, condition (3.1) is valid with $\beta=\frac{1}{2}$. We have $\sigma_{t k}^{2}=\sigma_{t \alpha_{k}}^{2}=q_{t}\left(\theta_{\alpha_{k}}\right)\left(1-q_{t}\left(\theta_{\alpha_{k}}\right)\right)$. Here

$$
q_{t}\left(\theta_{\alpha_{k}}\right)=\frac{b_{t} \theta_{\alpha_{k}}^{t}}{t ! B\left(\theta_{\alpha_{k}}\right)} \geq \frac{b_{t}\left(\theta^{\prime}\right)^{t}}{t ! B\left(\theta^{\prime \prime}\right)}>0
$$

as $b_{t}>0$. As $b_{0}>0, b_{1}>0$, we have $1-q_{t}\left(\theta_{\alpha_{k}}\right)>c>0$. So $\sigma_{t k}^{2} \geq c_{2}>0$ for each $k$, i.e. (3.2) is satisfied. So Theorem 4.1 follows from Theorem 3.1.

Let $\mu_{t}$ be the number of the random variables $\eta_{1}, \ldots, \eta_{N}$ being equal to $t, t=0,1, \ldots, n$. That is

$$
\mu_{t}=\mu_{t n N}=\sum_{i=1}^{N} \mathbb{I}_{\left\{\eta_{i}=t\right\}}
$$

Theorem 4.2. Let $0<\alpha^{\prime}<\alpha^{\prime \prime}$ be such that $m^{-1}\left(\alpha^{\prime \prime}\right)<R$. Let $t$ be fixed and assume that $b_{t}>0$. Then, for $\mu_{t n N}$ defined in (4.9), we have

$$
\limsup _{n, N \rightarrow \infty, \alpha^{\prime}<\alpha<\alpha^{\prime \prime}} \frac{\left|\mu_{t n N}-\mathbb{E} \mu_{t n N}\right|}{\sqrt{N \ln (N)} \sigma_{t \alpha}} \leq 2 \sqrt{10} \quad \text { almost surely. }
$$

Proof. First we remark that, for any two index sequence $l_{n, N}, \quad \lim \sup _{n, N \rightarrow \infty, \alpha^{\prime}<\alpha<\alpha^{\prime \prime}} l_{n, N}$ exists and it is unique because of $0<\alpha^{\prime}<\alpha^{\prime \prime}<\infty$. As $b_{t}>0$, and $b_{0}>0, b_{1}>0$, we have $\sigma_{t \alpha}^{2} \geq c_{1}>0$. Therefore for $B$ from $(2.2)$ we have $B=B\left(N, \sigma_{\alpha}\right) \leq K<\infty$ as $N, n \rightarrow \infty$ such that $\alpha^{\prime}<\alpha<\alpha^{\prime \prime}$.

Let $z>2 \sqrt{10}$. Then $\frac{z^{2}}{16}-\frac{1}{2}-1>1$. Therefore, by (2.1), we obtain

$$
\begin{gathered}
\sum_{N=N_{0}+1}^{\infty} \sum_{N \alpha^{\prime}<n<\alpha^{\prime \prime} N} \mathbb{P}\left\{\frac{\left|\mu_{t n N}-\mathbb{E} \mu_{t n N}\right|}{\sqrt{N \ln (N)} \sigma_{t \alpha}} \geq z\right\}= \\
=\sum_{N=N_{0}+1}^{\infty} \sum_{N \alpha^{\prime}<n<\alpha^{\prime \prime} N} \mathbb{P}\left\{\frac{\left|\mu_{t n N}-\mathbb{E} \mu_{t n N}\right|}{\sqrt{N}} \geq \sigma_{t \alpha} \sqrt{\ln (N)} z\right\} \leq \\
\leq\left(\sup _{\left\{N>N_{0}, \alpha^{\prime}<\alpha<\alpha^{\prime \prime}\right\}}(1+B)\right) \sum_{N=N_{0}+1}^{\infty} 4 \sqrt{2}\left(\left(\alpha^{\prime \prime}-\alpha^{\prime}\right) N\right) \sqrt{N} \sqrt{C_{2}} e^{-\frac{\ln (N) z^{2}}{16}} \leq \\
\leq\left(\sup _{\left\{N>N_{0}, \alpha^{\prime}<\alpha<\alpha^{\prime \prime}\right\}}(1+B)\right) 4 \sqrt{2} \sqrt{C_{2}}\left(\alpha^{\prime \prime}-\alpha^{\prime}\right) \sum_{N=N_{0}+1}^{\infty} N^{-\frac{z^{2}}{16}+\frac{1}{2}+1}<\infty .
\end{gathered}
$$

Consequently, by the Borel-Cantelli lemma, we have $\lim \sup _{n, N \rightarrow \infty, \alpha^{\prime}<\alpha<\alpha^{\prime \prime}} \frac{\left|\mu_{t n N}-\mathbb{E} \mu_{t n N}\right|}{\sigma_{t \alpha} \sqrt{N \ln (N)}} \leq z$ almost surely. This implies (4.10).

Corollary 4.1. Let $0<\alpha^{\prime}<\alpha^{\prime \prime}$ be such that $m^{-1}\left(\alpha^{\prime \prime}\right)<R$. Let $b_{t}>0$. Let $d_{N} \in \mathbb{R}, N \in \mathbb{N}$, be such that $\lim _{N \rightarrow \infty} \frac{\sqrt{N \ln (N)}}{d_{N}}=0$. Then, for $\mu_{t n N}$ defined in (4.9), we have

$$
\lim _{n, N \rightarrow \infty, \alpha^{\prime}<\alpha<\alpha^{\prime \prime}} \frac{\mu_{t n N}-\mathbb{E} \mu_{t n N}}{d_{N}}=0 \text { almost surely. }
$$

Proof. As $\sigma_{t \alpha} \leq C_{2}<\infty,(4.11)$ is an easy consequence of Theorem 4.2. 


\section{Applications to the nonuniform allocation scheme}

Consider the nonuniform scheme of allocations of $n$ distinguishable balls into $N$ boxes with nonuniform probabilities of boxes. The number of the balls in the boxes can be described with random variables $\eta_{1}, \ldots, \eta_{N}$ having polynomial distribution. As we shall consider $N \rightarrow \infty$, the distribution depends on $N$. So, for each $N$, we have random variables $\eta_{1}, \eta_{2}, \ldots, \eta_{N}$ with the distribution

$$
\mathbb{P}\left\{\eta_{1}=k_{1}, \eta_{2}=k_{2}, \ldots, \eta_{N}=k_{N}\right\}=\frac{n !}{k_{1} ! k_{2} ! \ldots k_{N} !}\left(p_{N 1}\right)^{k_{1}}\left(p_{N 2}\right)^{k_{2}} \ldots\left(p_{N N}\right)^{k_{N}}
$$

where $k_{1}+k_{2}+\ldots k_{N}=n, p_{N i}>0,1 \leq i \leq N$, and $p_{N 1}+p_{N 2}+\cdots+p_{N N}=1$.

Then the number of boxes which contain $s$ balls is

$$
\mu_{s n N}=\sum_{i=1}^{N} \mathbb{I}_{\left\{\eta_{i}=s\right\}}
$$

Let $\pi=\pi(\lambda)$ be a Poissonian random variable with parameter $\lambda$, i.e. $\pi(\lambda, k)=\mathbb{P}(\pi(\lambda)=$ $k)=\lambda^{k} e^{-\lambda} / k$ !. Let $\alpha=\alpha_{n, N}=\frac{n}{N}$. We will assume that $\alpha^{\prime} \leq \alpha_{n N} \leq \alpha^{\prime \prime}$ for some $0<\alpha^{\prime}<\alpha^{\prime \prime}<\infty$.

For the sequence of functions

$$
f_{N}(t)=N p_{N[N t]}, \quad t \in[0,1], \quad N \in \mathbb{N}
$$

we will consider the condition

$$
\lim _{N \rightarrow \infty} f_{N}(t) \rightarrow f(t)
$$

uniformly for $t \in[0,1]$. Here $f(t), t \in[0,1]$, is a bounded nonnegative function such that

$$
\int_{0}^{1} f(t) d t=1
$$

Let $\sigma_{n N i}^{2}$ be the variance of $\mathbb{I}_{\left\{\pi\left(n p_{N i}\right)=s\right\}}$. Consider their arithmetic mean, i.e. let

$$
\begin{gathered}
\bar{\sigma}_{n N}^{2}=\frac{1}{N} \sum_{i=1}^{N} \sigma_{n N i}^{2}=\frac{1}{N} \sum_{i=1}^{N} \pi\left(n p_{N i}, s\right)\left(1-\pi\left(n p_{N i}, s\right)\right)= \\
=\int_{0}^{1} \pi\left(\alpha f_{N}(t), s\right)\left(1-\pi\left(\alpha f_{N}(t), s\right)\right) d t
\end{gathered}
$$

Theorem 5.1. Let $0<\alpha^{\prime}<\alpha^{\prime \prime}$. Assume that (5.3) is satisfied. Then, for $\mu_{\text {snN }}$ defined in (5.2), we have

$$
\limsup _{n, N \rightarrow \infty, \alpha^{\prime}<\alpha<\alpha^{\prime \prime}} \frac{\left|\mu_{s n N}-\mathbb{E} \mu_{s n N}\right|}{\sqrt{N \ln (N)} \bar{\sigma}_{n N}^{2}} \leq 2 \sqrt{10} \text { almost surely. }
$$


Proof. The probability (5.1) we can be represented as the conditional probability

$$
\begin{gathered}
\mathbb{P}\left\{\eta_{1}=k_{1}, \ldots, \eta_{N}=k_{N}\right\}= \\
=\mathbb{P}\left\{\pi_{1}\left(p_{N 1}\right)=k_{1}, \ldots, \pi_{N}\left(p_{N N}\right)=k_{N} \mid \pi_{1}\left(p_{N 1}\right)+\cdots+\pi_{N}\left(p_{N N}\right)=n\right\}= \\
=\mathbb{P}\left\{\pi_{1}\left(n p_{N 1}\right)=k_{1}, \ldots, \pi_{N}\left(n p_{N N}\right)=k_{N} \mid \pi_{1}\left(n p_{N 1}\right)+\cdots+\pi_{N}\left(n p_{N N}\right)=n\right\}
\end{gathered}
$$

where for each fixed $N,\left\{\pi_{i}\left(p_{N i}\right): 1 \leq i \leq N\right\}$ and $\left\{\pi_{i}\left(n p_{N i}\right): 1 \leq i \leq N\right\}$ are two families of independent Poissonian random variables.

We know that $\pi_{1}\left(n p_{N 1}\right)+\pi_{2}\left(n p_{N 2}\right)+\cdots+\pi_{N}\left(n p_{N N}\right)$ is a Poissonian random variable with parameter $n$. Therefore, by the Stirling formula, we have

$$
\mathbb{P}(A)=\mathbb{P}\{\pi(n)=n\}=e^{-n} \frac{n^{n}}{n !}=\frac{1}{\sqrt{2 \pi n}}(1+o(1)) \geq \frac{1}{\sqrt{2 \pi \alpha^{\prime \prime} N}}(1+o(1)) .
$$

Therefore (3.1) is valid with $\beta=\frac{1}{2}$. From the uniform convergence (5.3) it follows that $\lim _{N \rightarrow \infty}\left|\bar{\sigma}_{n N}-\bar{\sigma}_{\alpha}\right|=0$ uniformly in $\alpha^{\prime} \leq \alpha \leq \alpha^{\prime \prime}$ where

$$
\bar{\sigma}_{\alpha}^{2}=\int_{0}^{1} \pi(\alpha f(t), s)(1-\pi(\alpha f(t), s)) d t .
$$

Here $\bar{\sigma}_{\alpha}$ is a continuous positive function of $\alpha \in\left[\alpha^{\prime}, \alpha^{\prime \prime}\right]$. Therefore $\inf _{\alpha^{\prime} \leq \alpha \leq \alpha^{\prime \prime}} \bar{\sigma}_{\alpha}^{2}=C_{4}>0$. So we can find $N_{0}$ such that for all $N>N_{0}$ and $\alpha^{\prime} \leq \alpha \leq \alpha^{\prime \prime}$ we have $\bar{\sigma}_{n N}^{2} \geq \frac{C_{4}}{2}>0$. So the proof can be finished as the proof of Theorem 4.2.

Corollary 5.1. Let $0<\alpha^{\prime}<\alpha^{\prime \prime}$. Let $b_{N} \in \mathbb{R}, N \in \mathbb{N}$, be such that $\lim _{N \rightarrow \infty} \frac{\sqrt{N \ln (N)}}{b_{N}}=0$. Suppose that (5.3) is valid. Then, for $\mu_{\text {snN }}$ defined in (5.2), we have

$$
\lim _{n, N \rightarrow \infty, \alpha^{\prime}<\alpha<\alpha^{\prime \prime}} \frac{\mu_{s n N}-\mathbb{E} \mu_{s n N}}{b_{N}}=0 \text { almost surely. }
$$

Consider a sequence of allocations of $n_{k}$ balls into $N_{k}$ boxes, $k \in \mathbb{N}$, such that $N_{k}<N_{k+1}$, $k \in \mathbb{N}$. Let $\alpha_{k}=n_{k} / N_{k}, \sigma_{s k}^{2}=\bar{\sigma}_{n_{k} N_{k}}^{2}$ and

$$
\mu_{s k}=\sum_{i=1}^{N_{k}} \mathbb{I}_{\left\{\eta_{i}=s\right\}}
$$

Theorem 5.2. Let $0<\alpha^{\prime}<\alpha^{\prime \prime}<\infty$. Let (5.3) be valid. Suppose that $0<\alpha^{\prime} \leq \alpha_{k} \leq \alpha^{\prime \prime}$ for all $k \in \mathbb{N}$. Then for $\mu_{\text {snN }}$ defined in (5.4), we have

$$
\limsup _{k \rightarrow \infty} \frac{\left|\mu_{s k}-\mathbb{E} \mu_{s k}\right|}{\sqrt{N_{k} \ln \left(N_{k}\right)} \sigma_{s k}} \leq 4 \sqrt{3 / 2} \text { almost surely. }
$$

Proof. From the proof of Theorem 5.1 it follows that (3.1) is valid with $\beta=\frac{1}{2}$. Also the proof of Theorem 5.1 implies that (3.2) is valid, too. So Theorem 5.2 follows from Theorem 3.1 . 
Theorem 5.3. Let $0<\alpha_{0}<\infty$. Assume that (5.3) is satisfied. Then, for $\mu_{s n N}$ defined in (5.2), we have

$$
\frac{1}{N} \mu_{s n N} \rightarrow \frac{\alpha_{0}^{s}}{s !} \int_{0}^{1}(f(t))^{s} e^{-f(t) \alpha_{0}} d t
$$

almost surely as $n, N \rightarrow \infty$ such that $\alpha \rightarrow \alpha_{0}$.

Proof. Let $\alpha^{\prime}<\alpha_{0}<\alpha^{\prime \prime}$. If $n, N \rightarrow \infty$ such that $\alpha \rightarrow \alpha_{0}$, then there exists $N_{0} \in \mathbb{N}$ such that $\alpha^{\prime}<\alpha<\alpha^{\prime \prime}$ for $n, N>N_{0}$. By Theorem 5.1, $\frac{\mu_{s n N}-\mathbb{E} \mu_{s n N}}{N} \rightarrow 0$ almost surely, as $n, N \rightarrow \infty$ such that $\alpha \rightarrow \alpha_{0}$. Furthermore, we have

$$
\begin{gathered}
\frac{1}{N} \mathbb{E} \mu_{s n N}=\frac{1}{N} \sum_{k=1}^{N} \frac{n !}{s !(n-s) !}\left(p_{N k}\right)^{s}\left(1-p_{N k}\right)^{n-s}= \\
=\frac{1}{s !} \frac{1}{N} \sum_{k=1}^{N} \frac{n}{N} \cdot \frac{n-1}{N} \ldots \frac{n-s+1}{N}\left(N p_{N k}\right)^{s}\left(1-\frac{N p_{N k}}{N}\right)^{n-s}=\int_{0}^{1} g_{n N}\left(f_{N}(t)\right) d t .
\end{gathered}
$$

Here

$$
\begin{aligned}
g_{n N}\left(f_{N}(t)\right)=\frac{1}{s !} & \frac{n}{N} \cdot \frac{n-1}{N} \ldots \frac{n-s+1}{N}\left(f_{N}(t)\right)^{s}\left(1-\frac{f_{N}(t)}{N}\right)^{n-s} \approx \\
& \approx \frac{\alpha^{s}}{s !}\left(f_{N}(t)\right)^{s}\left(1-\frac{f_{N}(t)}{N}\right)^{N \alpha} .
\end{aligned}
$$

So $0 \leq g_{n N}\left(f_{N}(t) \leq C\right.$ for all $t \in[0,1]$. Moreover, $g_{n N}\left(f_{N}(t)\right) \rightarrow \frac{\left(\alpha_{0} f(t)\right)^{s}}{s !} e^{-f(t) \alpha_{0}}$ as $n, N \rightarrow \infty$ such that $\alpha \rightarrow \alpha_{0}$ for all $t \in[0,1]$. Therefore, by the Lebesgue theorem, it holds that

$$
\frac{1}{N} \mathbb{E} \mu_{s n N} \rightarrow \frac{\alpha_{0}^{s}}{s !} \int_{0}^{1}(f(t))^{s} e^{-f(t) \alpha_{0}} d t
$$

as $n, N \rightarrow \infty$ such that $\alpha \rightarrow \alpha_{0}$. Consequently,

$$
\frac{1}{N} \mu_{s n N}=\frac{1}{N} \mu_{s n N}-\frac{1}{N} \mathbb{E} \mu_{s n N}+\frac{1}{N} \mathbb{E} \mu_{s n N} \rightarrow \frac{\alpha_{0}^{s}}{s !} \int_{0}^{1}(f(t))^{s} e^{-f(t) \alpha_{0}} d t
$$

as $n, N \rightarrow \infty$ such that $\alpha \rightarrow \alpha_{0}$. The proof is complete.

Consider the number of boxes containing less than $r$ balls and consider also the number of boxes containing more than $r$ balls, i.e. let

$$
\mu_{<r N}=\mu_{<r n N}=\sum_{i=1}^{N} \mathbb{I}_{\left\{\eta_{i}<r\right\}}, \quad \mu_{>r N}=\mu_{>r n N}=\sum_{i=1}^{N} \mathbb{I}_{\left\{\eta_{i}>r\right\}} .
$$

Since $\mu_{<r n N}=\sum_{k=0}^{r-1} \mu_{k n N}$ and $\mu_{>r n N}=N-\mu_{<r n N}-\mu_{r n N}$, therefore Theorem 5.3 implies the following. 
Corollary 5.2. Let $0<\alpha_{0}<\infty$. Suppose that (5.3) is valid. Then we have

$$
\frac{1}{N} \mu_{<r n N} \rightarrow \sum_{k<r} \frac{\alpha_{0}^{k}}{k !} \int_{0}^{1}(f(t))^{k} e^{-f(t) \alpha_{0}} d t
$$

and

$$
\frac{1}{N} \mu_{>r n N} \rightarrow \sum_{k>r} \frac{\alpha_{0}^{k}}{k !} \int_{0}^{1}(f(t))^{k} e^{-f(t) \alpha_{0}} d t
$$

almost surely, as $n, N \rightarrow \infty$ such that $\alpha \rightarrow \alpha_{0}$.

Consider the number of boxes such that the numbers of balls contained by these boxes belong to the subset $\mathbb{N}^{\prime} \subset \mathbb{N}$, i.e. let

$$
\mu_{\mathbb{N}^{\prime} N}=\mu_{\mathbb{N}^{\prime} n N}=\sum_{i=1}^{N} \mathbb{I}_{\left\{\eta_{i} \in \mathbb{N}^{\prime}\right\}}
$$

Corollary 5.3. Let $0<\alpha_{0}<\infty$. Suppose that (5.3) is valid. Then we have

$$
\frac{1}{N} \mu_{\mathbb{N}^{\prime} n N} \rightarrow \sum_{k \in \mathbb{N}^{\prime}} \frac{\alpha_{0}^{k}}{k !} \int_{0}^{1}(f(t))^{k} e^{-f(t) \alpha_{0}} d t
$$

almost surely, as $n, N \rightarrow \infty$ such that $\alpha \rightarrow \alpha_{0}$.

Proof. First let $\mathbb{N}^{\prime}$ be a finite set. Since $\mu_{\mathbb{N}^{\prime} n N}=\sum_{k \in \mathbb{N}^{\prime}} \mu_{k n N}$, by Theorem 5.3, (5.5) is true. Now, let $\mathbb{N}^{\prime}$ be an arbitrary set. Denote $\mathbb{N}_{r}^{\prime}=\left\{k \in \mathbb{N}^{\prime}: k \leq r\right\}$. Then we have

$$
\mu_{\mathbb{N}_{r}^{\prime} N} \leq \mu_{\mathbb{N}^{\prime} N} \leq \mu_{\mathbb{N}_{r}^{\prime} N}+\mu_{>r N}
$$

Therefore

$$
\begin{gathered}
\sum_{k \in \mathbb{N}_{r}^{\prime}} \frac{\alpha_{0}^{k}}{k !} \int_{0}^{1}(f(t))^{k} e^{-f(t) \alpha_{0}} d t \leq \liminf \frac{1}{N} \mu_{\mathbb{N}^{\prime} N} \leq \limsup \frac{1}{N} \mu_{\mathbb{N}^{\prime} N} \leq \\
\sum_{k \in \mathbb{N}_{r}^{\prime}} \frac{\alpha_{0}^{k}}{k !} \int_{0}^{1}(f(t))^{k} e^{-f(t) \alpha_{0}} d t+\sum_{k>r} \frac{\alpha_{0}^{k}}{k !} \int_{0}^{1}(f(t))^{k} e^{-f(t) \alpha_{0}} d t .
\end{gathered}
$$

almost surely. Let $r \rightarrow \infty$, then (5.6) implies (5.5). The proof is complete.

\section{References}

[1] Chuprunov, A. and Fazekas, I. Inequalities and strong laws of large numbers for random allocations. Acta Math. Hungar. 109 (2005), no. 1-2, 163-182. 
[2] Chuprunov, A. and Fazekas, I. Strong laws of large numbers for random forests. Acta Math. Hungar. 124 (2009), no. 1-2, 59-71.

[3] Chuprunov, A. and Fazekas, I. An inequality for moments and its applications to the generalized allocation scheme. Publ. Math. Debrecen, 76 (2009), no. 3-4, 271-286.

[4] Haagerup, U. The best constants in the Khintchine inequality. Studia Math. 70 (1981), no. 3, 231-283 (1982).

[5] Kolchin V.F. Random Graphs, Cambridge University Press, Cambridge, 1999.

[6] Kolchin, A. V. Limit theorems for a generalized allocation scheme. (Russian) Diskret. Mat. 15 (2003), no. 4, 148-157; translation in Discrete Math. Appl. 13 (2003), no. 6, $627-636$.

[7] Kolchin, V.F., Sevast'yanov, B.A. and Chistyakov, V.P. Random allocations. V.H. Winston \& Sons, Washington D. C., 1978.

[8] Khintchine, A. Über dyadische Brüche. Math. Zeitschr. 18 (1923), 109-116.

[9] Móri, T. F. Sharp inequalities between centered moments. J. Inequal. Pure Appl. Math. 10 (2009), no.4.

[10] Nemes, G. New asymptotic expansion for the $\Gamma(x)$ function. Stan's Library, December 7, 2008, http://dx.doi.org/10.3247/sl2math08.005

[11] Pavlov, Yu. L. Random Forests. VSP, Utrecht, 2000.

[12] Rényi, A. Three new proofs and generalization of a theorem of Irving Weiss. Magy. Tud. Akad. Mat. Kutató Int. Közl. 7(1-2) (1962), 203-214.

[13] Weiss, I. Limiting distributions in some occupancy problems. Ann. Math. Statist. 29(3) (1958), 878-884. 